\title{
Reconstruction of Midfacial Defects Following Surgical Management of Skin Cancer
}

\author{
The Role of Tissue Expansion
}

\author{
SHAN R. BAKER, MD \\ NEIL A. SWANSON, MD
}

\begin{abstract}
Compared with other locations on the face, skin cancer of the midface has a higher risk of recurrence owing, in part, to the mode of spread and the high frequency of subclinical extension. Skin cancers occurring in the region of the melolabial crease, nasal columella and medial canthus should be resected by Mohs surgery. Because tumors of the midface are sometimes recalcitrant, there are times when surgical ablation results in extensive defects. Reconstruction may be difficult owing to lack of available donor tissue, or because of previous surgery in the area of the proposed donor region. In these circumstances, tissue expansion in the form of controlled prolonged expansion or rapid intraoperative expansion, may, on occasion, provide the optimal reconstruction of form and function. J Dermatol Surg Oncol 1994;20:133-140.
\end{abstract}

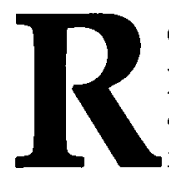
econstruction of the midface is a challenging problem because of complex topographical anatomy, as well as the vital functions performed by the facial structures within this region. The midface includes the upper lip, entire nose, central forehead and medial canthal region. Most defects of the midface that require reconstruction result from the need to excise skin cancers. Skin cancer is the most common form of malignancy in man, and the midface is the most common location. ${ }^{1}$ Basal cell carcinoma (BCC) is the predominant histological type. Fortunately, the mortality rate from $\mathrm{BCC}$ is negligible, but its morbidity can be significant when such tumors involve midface structures and are not treated early and properly. In the case of BCC, tumor histology is correlated with the frequency of recur-

From the Section of Facial Plastic and Reconstructive Surgery (SRB), Department of Otolaryngology, University of Michigan, Ann Arbor, Michigan, and the Departments of Dermatology, Otolaryngology and Plastic Surgery, (NAS), Oregon Health Science University.

Address correspondence and reprint requests to: Shan $R$. Baker, MD, 1500 E. Medical Center Drive, Ann Arbor, MI 48109-0312. rence; however, the site of the tumor appears to be a far more important factor in predicting treatment failure. ${ }^{1}$ The midface is the location of the greatest risk of recurrent basal cell carcinoma. ${ }^{1}$ This risk is highest when tumor is managed by curettage and electrodessication, and lowest when treated with surgical excision with complete histological control of the margins.

Basal cell carcinoma of the midface is difficult to treat in part because tumors of ten approach certain anatomical structures and spread along them before invading. This results in subclinical extension which is not always appreciated on examination. Specific examples of this phenomenon in the midface are tumor spread along the periosteum of nasal bones, perichondrium of alar cartilages, and the tarsal plate of the eyelids. Tumors may also spread along nerve sheaths and blood vessels by invasion of the perineural sheath and adventitia of arteries in the area.

Another mode of spread of BCC is along embryonic fusion planes. Tumors arising in areas of fusion planes may invade to an unexpected depth because these planes extend in a direction perpendicular to the surface of the skin. Important sites in the midface where this occurs are along the columella and septum of the nose and at the junction of the nasal alar base with the melolabial fold.

The algorithm of Figure 1 summarizes the author's approach to management of $\mathrm{BCC}$ of the midface. ${ }^{1}$ The majority of primary tumors occurring in the face may be treated with conventional methods; however, BCC occurring in high risk locations of the midface, specifically the melolabial fold, nasal columella and the region of the medial canthus should be resected by Mohs micrographic surgery. If this expertise is not available in the community, then such tumors should be managed by frozen section control of the periphery and base of the wound following surgical excision. Likewise, tumors having morpheaform or basosquamous histology, greater than $2 \mathrm{~cm}$ in size, having an aggressive growth pattern or multi-centric in origin should be treated in a similar man- 


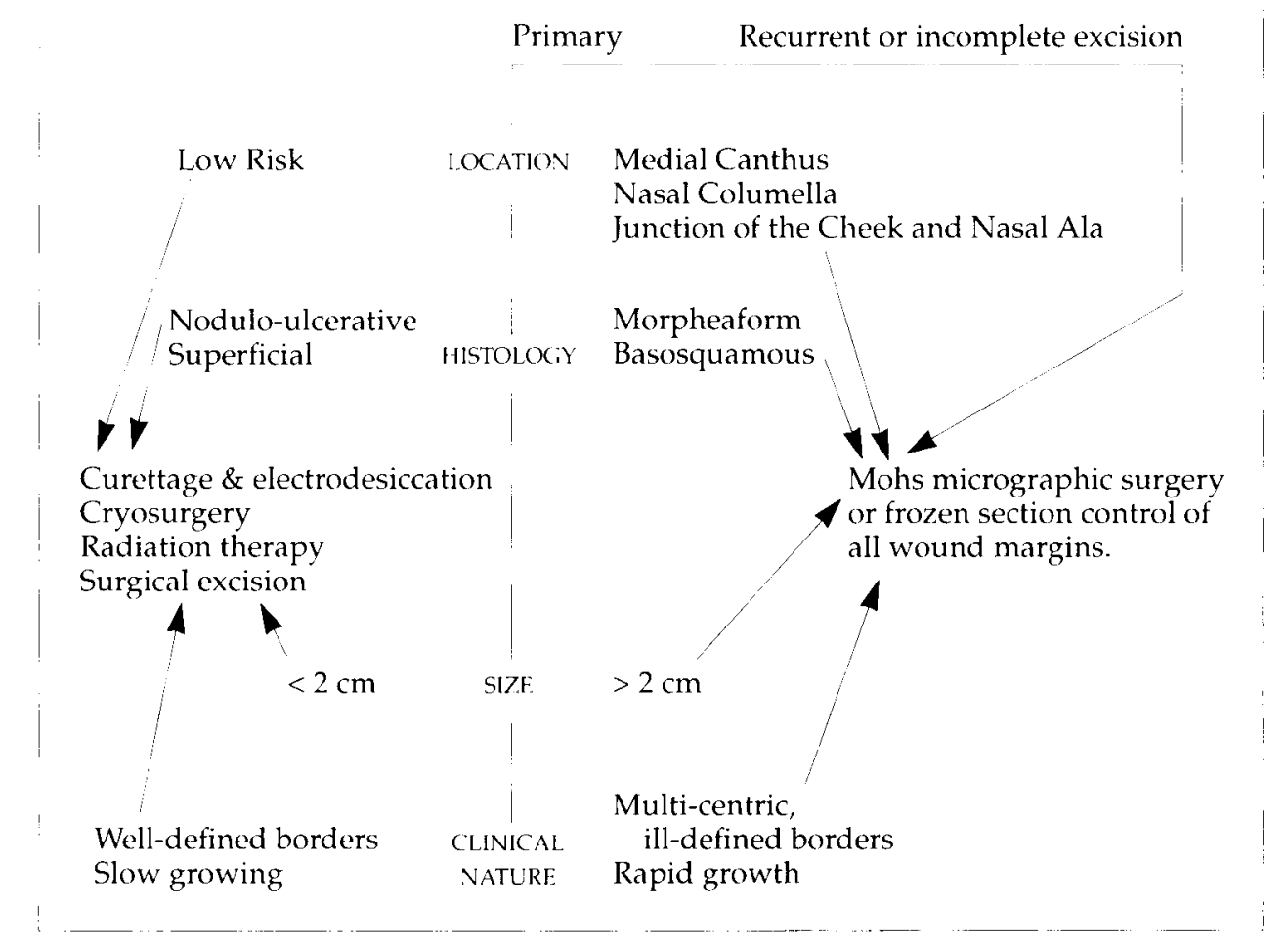

Figure 1. Management of midfacial basal cell carcinoma.

ner consisting of a detailed histological analysis of the resection margins.

\section{Reconstruction of Midfacial Skin Defects}

The algorithm in Figure 2 is an attempt to integrate form and function into a logical approach for formulating a method of repairing general surface defects of the midface. ${ }^{2}$ The first consideration of the clinician is a choice of repair by primary closure of the wound or use of a skin graft. Occasionally, primary closure may not be feasible and a skin graft can be used without unduly affecting form and function. Larger defects by necessity may frequently require alternative methods of reconstruction. The next echelon in the order of thinking is the consideration of local flaps. Similar to primary closure or the use of skin grafts, the detriment of form and function resulting from the use of local flaps must be considered. When the detriment is excessive, consideration must be given to the use of regional flaps. This natural progression from simple to more complex methods of reconstruction provides a systematic approach for evaluating any combination of defects of the midface. Each step in the algorithm is gov- erned by the experience and clinical judgment of the person responsible for reconstruction.

Most defects of the midface are preferably reconstructed with adjacent local skin flaps if primary wound repair is not possible. Unfortunately, there are times when the reconstructive surgeon is confronted with midfacial defects resulting from ablating extensive skin cancers that are difficult or impossible to repair with local flaps. Although regional, distant, or microsurgical flaps are always a reconstructive option, the marked discrepancy in the texture and color of the skin of these flaps compared to the facial skin causes these options to be less desirable. The undesirability is enhanced by the greater complexity of transferring such flaps to the face. There are also situations where a midfacial defect is not particularly large, but the use of a local flap is made more difficult because of previous surgery in the area. The situations described above are ones where the authors have found tissue expansion utilized as either a one-stage immediate intraoperative expansion or two-staged prolonged controlled expansion to be extremely helpful in providing an optimal reconstruction, both in form and function.

Tissue expansion is indicated in the reconstruction of 


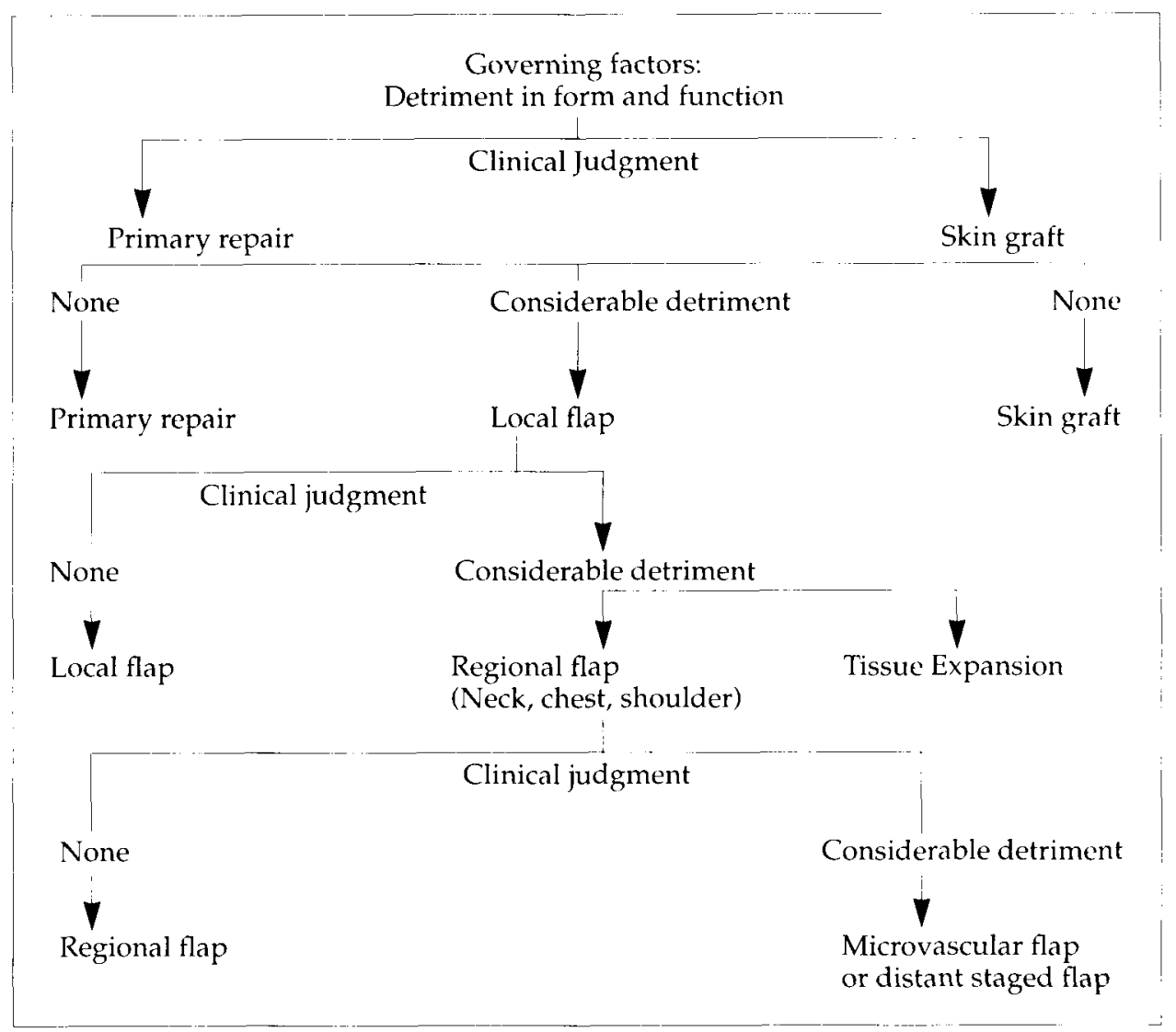

Figure 2. Options for reconstruction of surface defects of the midface.

various defects of the midface and orbit in instances where there is inadequate adjacent tissue to allow either primary closure of the defect or repair with a local flap., Its use may also be indicated for reconstruction of a defect that can be repaired by local, regional or distant flaps, but at the cost of significant donor or recipient site deformity. Indeed, there are times when tissue expansion is the preferred method of reconstructing midfacial defects because it can provide the most optimal results when compared with other reconstructive options.

It is not the purpose of this article to discuss the techniques of tissue expansion. The authors have published a detailed description of their methodology in the recent past. ${ }^{3,4}$ However, for the sake of providing the dermatologist who may not be familiar with the mechanics of expander insertion and inflation, a brief summary is provided: Tissue expanders used in the head and neck are implanted under local anesthesia following ringed block using $1 \%$ lidocaine and epinephrine $(1: 100,000)$. In the case of the scalp or forehead, a recipient pocket is created between the periosteum and the galea or deep fascia of the frontalis muscle, respectively. The pocket should be of sufficient size to allow the base of the expander to lie flat without folding or distortion. The injection port should be placed through the same incision as that of the expander, but situated in a separate pocket approximately $6 \mathrm{~cm}$ away from the balloon. Meticulous hemostasis must be achieved, since drains are not used. The expander is partially expanded with saline before wound closure to concomitantly obliterate dead space and assist with hemostasis. The incision is closed by layers using permanent suture for approximation of both the subcutaneous as well as the cutaneous tissues.

Inflation begins 2 weeks after implantation of the expander. Saline is infused by percutaneous puncture of the injection port with a 23 -guage scalp needle attached to a $50-\mathrm{mL}$ volume syringe after preparing the injection site with an alcohol swab. Some children may require anesthetizing the skin overlying the injection port before inflation. The volume of injection depends on the tensile strength and tension of the skin overlying the expander and the amount of patient discomfort. If not precluded by discomfort, the tissue should be expanded until slight blanching is observed in the skin overlying the expander. 
Saline should then be withdrawn until the blanching disappears. Inflation is usually conducted as frequently as once or twice a week. More rapid expansion may be possible, but is associated with a greater risk of expander extrusion. Periodic expansion continues until the circumference of the dome of the expanded skin measures two or three times the width of the proposed defect. This usually takes 6 to 8 weeks for the skin of the forehead and neck and up to 12 weeks for the scalp. The second surgical stage involves removal of the expander and proceeding with reconstruction.

The subsequent portions of this article will present a few examples where tissue expansion was helpful in reconstructing midfacial defects that presented as surgical dilemmas due to the lack of donor sites for local flaps or because of the extensiveness of the defect.

\section{Case Reports}

\section{Case 1}

A 71-year-old male presented with a recurrent aggressive $\mathrm{BCC}$ involving the right medial canthus, nasal dorsum and glabellar and forehead areas. This tumor had been treated several times in the past, including full course radiotherapy and Mohs fixed tissue technique on one occasion, as well as Mohs fresh tissue technique on another occasion. The patient underwent Mohs excision of the recurrent tumor, requiring five stages. The tumor was found to involve the frontal bone in the glabellar area and the nasal bony dorsum. Because of the extensive bone involvement, it was necessary to perform a frontal sinusectomy, removing the entire anterior and inferior frontal sinus walls and both nasal bones. Bilateral external ethmoidectomies were also performed, leaving a bony and soft tissue defect measuring $5 \times 7 \mathrm{~cm}$ (Figure 3). Portions of the defect were covered with a split-thickness skin graft. It was elected to postpone reconstruction for 18 months because of the extensiveness of the neoplasm and the concern for possible recurrence. A nasal prosthesis was fashioned to cover the nose and forehead defect.

This patient presented a reconstructive challenge because of the large defect that involved loss of both supratrochlear arteries and central forehead skin, precluding the use of a median or paramedian forehead flap, which would have been the preferred source of local tissue for reconstruction of this large defect. A scalping forehead flap would have been a reconstructive option; however, such a procedure usually requires the use of a split-thickness skin graft to repair the donor site, leaving an unsightly deformity of the lateral forehead. The resection of portions of forehead skin also place some constraints on other types of forehead flaps that might have been uti- lized. Controlled prolonged tissue expansion offered an opportunity to circumvent the lack of donor tissue by expanding lateral forehead skin sufficiently to provide tissue for near total nasal reconstruction, repair of the midline forehead skin defect, and, at the same time, allow primary closure of the donor site. A transversely oriented forehead flap based on the blood supply of the supra-orbital vessels was utilized following expansion of the lateral forehead skin over an 8-week period, using a $250-\mathrm{mL}$ rectangular expander (Figure 3). The internal lining for the reconstructed upper nasal passages was provided for by placing a split-thickness skin graft on the deep surface of the transposed flap in the area of the superior one third of the nose.

This case represents an excellent example in which controlled prolonged tissue expansion enabled reconstruction of a defect with local tissue and primary closure of the donor site in a situation which, without tissue expansion, would not have been possible. Tissue expansion offered the opportunity to optimize the cosmetic result in a situation where the preferred method of reconstruction using a midline forehead flap was not possible because of loss of skin and vascularity of the central forehead region. Unfortunately, the patient died of causes unrelated to his skin cancer or reconstructive surgery approximately 2 months following the transposition of the expanded forehead flap. This precluded subsequent release of the flap and insertion of skeletal support for the dorsum.

\section{Case 2}

A 62-year-old female presented with a 1-year history of a growth involving the nasal tip and dorsum. Biopsy confirmed the presence of a BCC which was treated with Mohs surgery. It was necessary to remove all of the left lower lateral nasal cartilage; fortunately, however, the alar bases were preserved. In addition, most of the upper lateral cartilages were resected bilaterally, leaving a fullthickness tip and nasal dorsal defect. The defect measured approximately $4 \times 5$ centimeters, and extended onto the left medial cheek (Figure 4).

A midline forehead flap was the preferred method of reconstruction; however, the size of the defect would have required a forehead flap of such dimensions that primary closure of the forehead would have been impossible. The exposed nasal bones and medial cheek defect were covered with a split-thickness skin graft and a tissue expander was inserted beneath the forehead skin in anticipation of performing expansion of the region prior to reconstruction with a midline forehead flap. Controlled prolonged expansion was accomplished over an 8-week period with weekly partial inflations of a $250-\mathrm{mL}$ rectangular expander. Figure 4 demonstrates the degree of ex- 


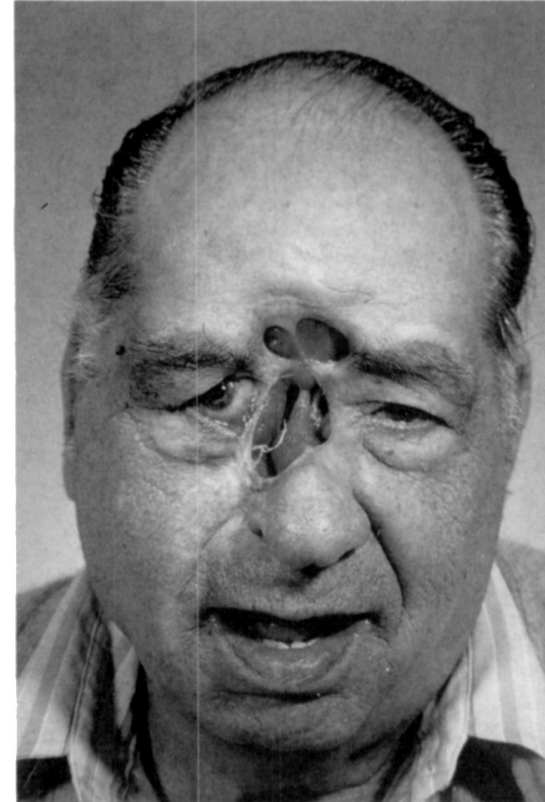

A

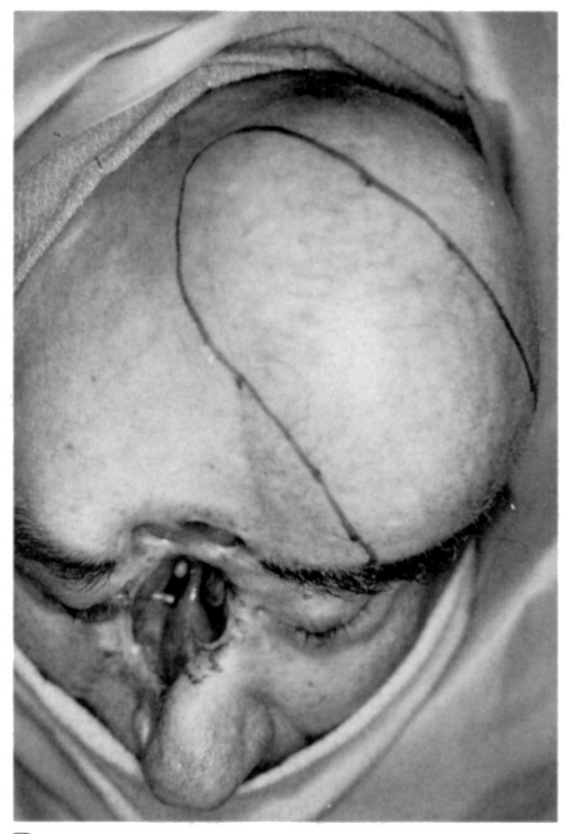

B

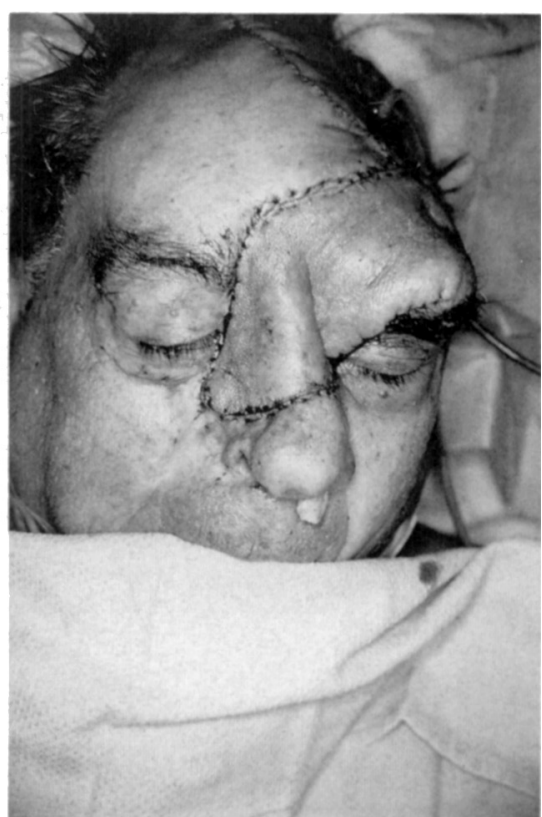

C

Figure 3. A) Defect of midface following removal of extensive basal cell carcinoma. It was necessary to remove the anterior and inferior walls of the frontal sinus, as well as the forehead skin overlying the sinus. B) Transversely oriented flap based on the supraorbital artery and vein is designed to center over the expander. C) Forehead flap transposed. Skin expansion provided sufficient tissue to reconstruct entire defect and enabled primary closure of the donor site.

pansion achieved just prior to reconstructive surgery. Ample skin was available for near total nasal reconstruction and for primary closure of the forehead donor site.

This case represents a surgical dilemma of less complexity than encountered in Case 1 . In this situation, reconstruction would have been possible using a midline forehead flap in a one-stage procedure, but it would not have been possible to close completely the donor site primarily. This approach would have resulted in a less than optimal cosmetic result.

\section{Case 3}

A 69-year-old man with diabetes mellitus presented with a large recurrent basal cell carcinoma of the left nasal dorsum which had been treated previously on two separate occasions (Figure 5). The patient underwent Mohs excision of the tumor, resulting in a dorsal nasal skin defect measuring approximately $4 \mathrm{~cm}$ in greatest width. A midline forehead flap was the preferred method of reconstruction; however, the patient's forehead skin was taut, and primary closure of the forehead skin following transposition of a $4-\mathrm{cm}$ wide midline forehead flap would have been difficult, causing excessive wound tension along the entire length of the donor site. Intraoperative rapid tissue expansion was performed to facilitate wound closure. A 30-mL tissue expander was used to expand the forehead skin prior to transposition of the flap. Three expansions, lasting 3 minutes each with 3 -minute rest intervals between expansions, were utilized. Rapid intraoperative tissue expansion allowed stretching of a $3.5-\mathrm{cm}$ wide flap to $4.2 \mathrm{~cm}$, as well as stretching the surrounding skin, which facilitated wound closure without tension following flap transfer (Figure 5).

This case represents the least complex surgical challenge of the three cases presented. In contrast to the first two cases, in this case, sufficient tissue was available to reconstruct the midfacial defect with local tissue and primary closure of the donor site was possible, but perhaps at the expense of excessive wound tension. This may have resulted in a wider forehead scar. Rapid intraoperative tissue expansion helped reduce this wound tension and presumably assisted in improving the cosmetic appearance of the donor site.

In contrast to prolonged controlled tissue expansion, which is limited primarily to elective reconstructive situations that require the generation of significant additional skin, rapid intraoperative tissue expansion can be used at a moment's notice and permits the stretching of skin beyond its non-inherant limits within minutes. The authors have, in the past, published a description of the techniques of rapid intraoperative tissue expansion.,6

Controlled, prolonged skin expansion produces larger surface area gains than rapid intraoperative expansion. 


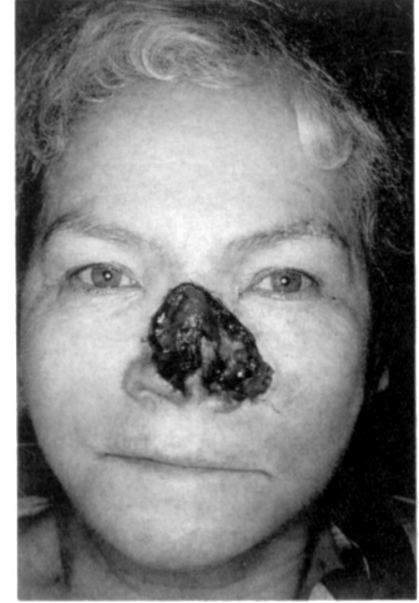

A

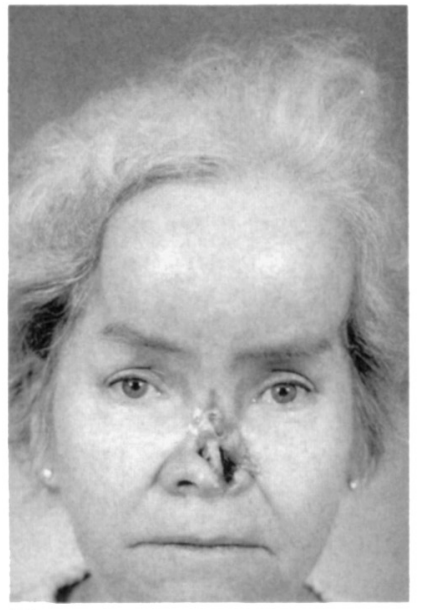

C

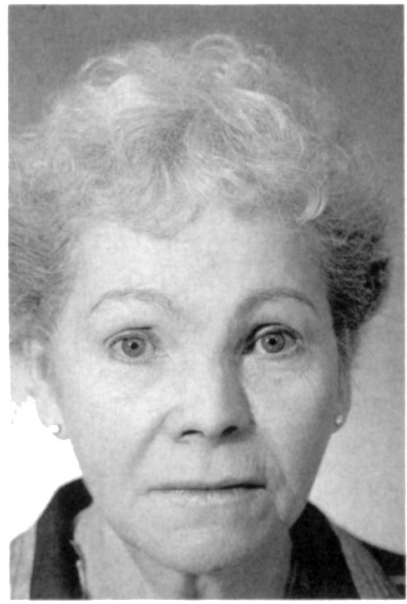

E

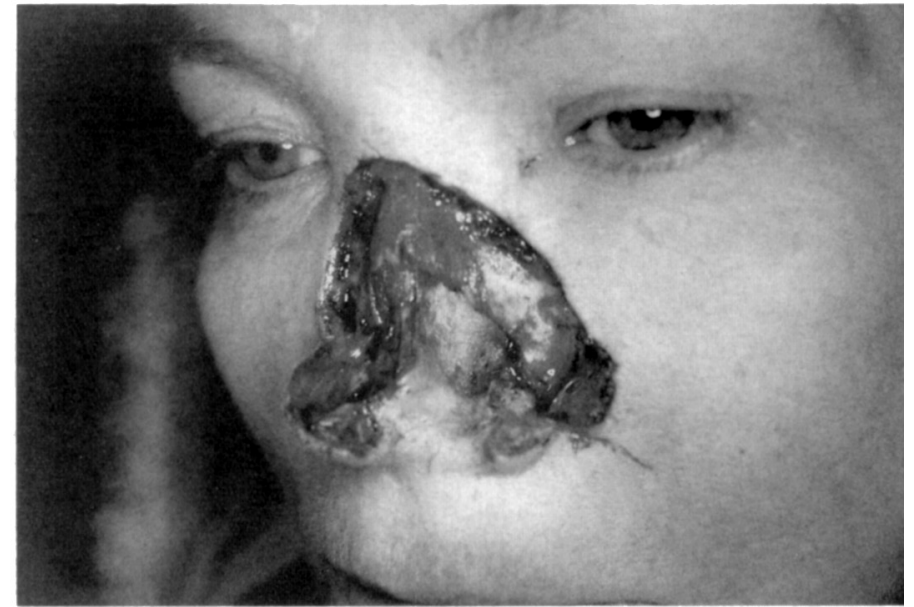

B

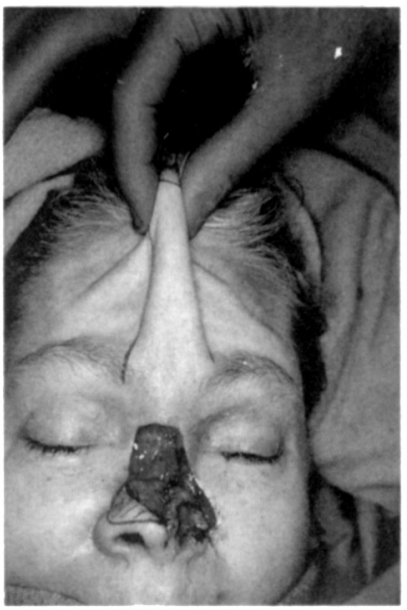

D

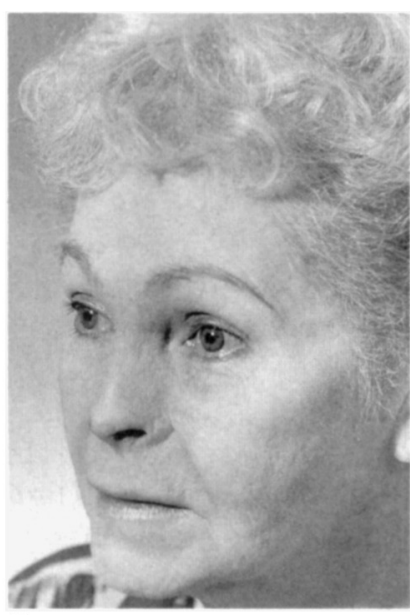

F midfacial defect following excision of a basal cell carcinoma. The majority of the left nasal tip and ala were resected, as well as both upper lateral cartilages, leaving a full thickness tip and nasal dorsal defect measuring approximately $4.0 \times 5.0$ centimeters. C) A $250 \mathrm{cc}$ volume rectangular expander was used to expand the entire forehead. $D$ ) Ample skin is observed immediately following decompression of the expander as a preliminary step to reconstruction. E and $\mathrm{F}$ Appearance of forehead and reconstructed nose 1 year following surgery.
Figure 4. $A$ and B) Extensive 


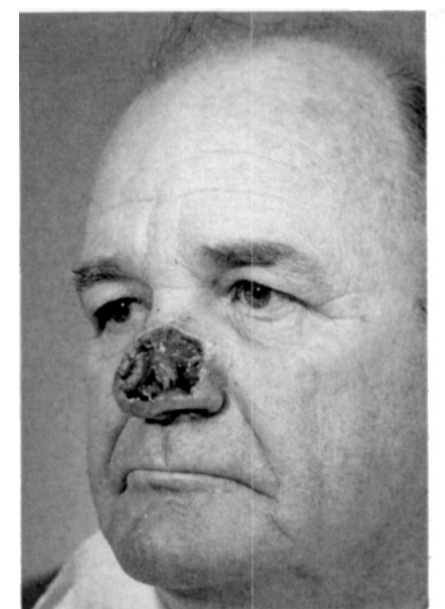

A
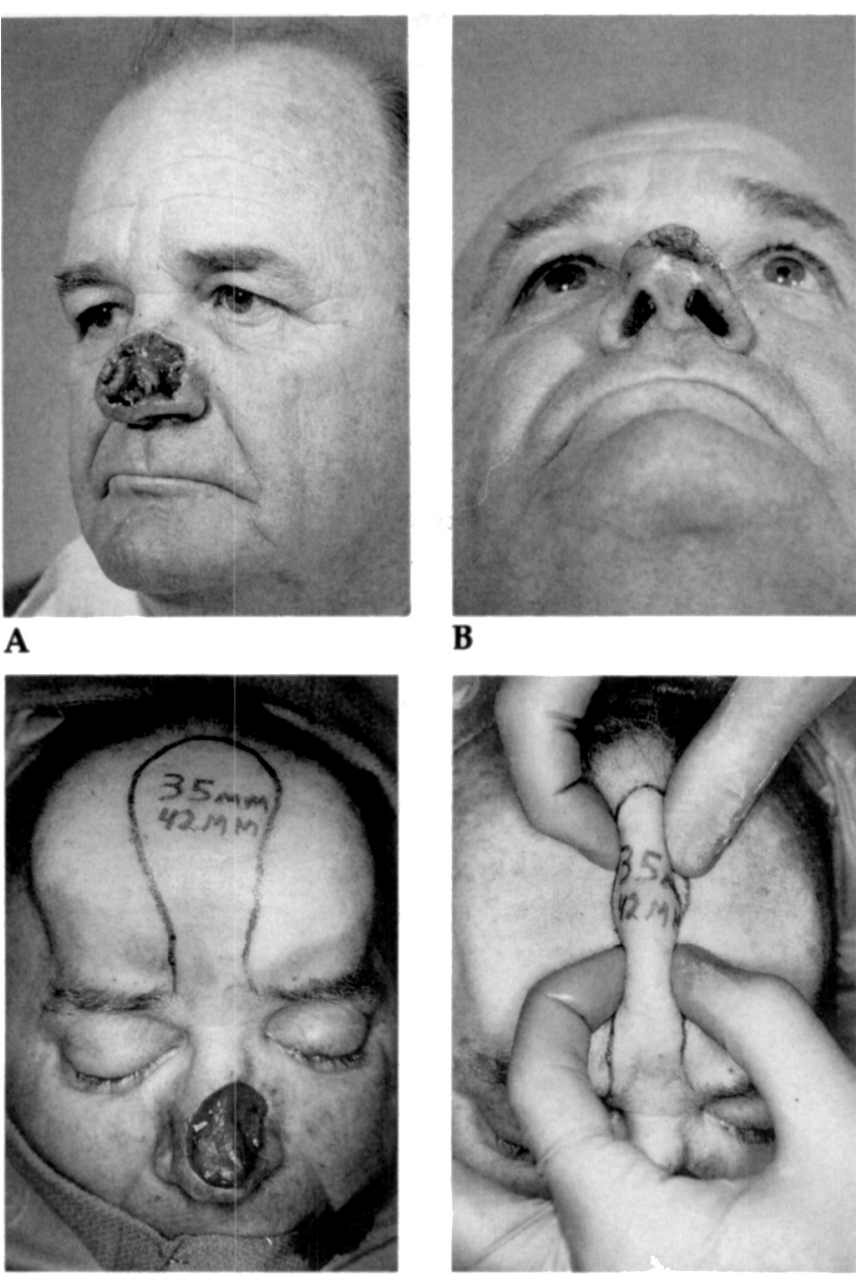

C
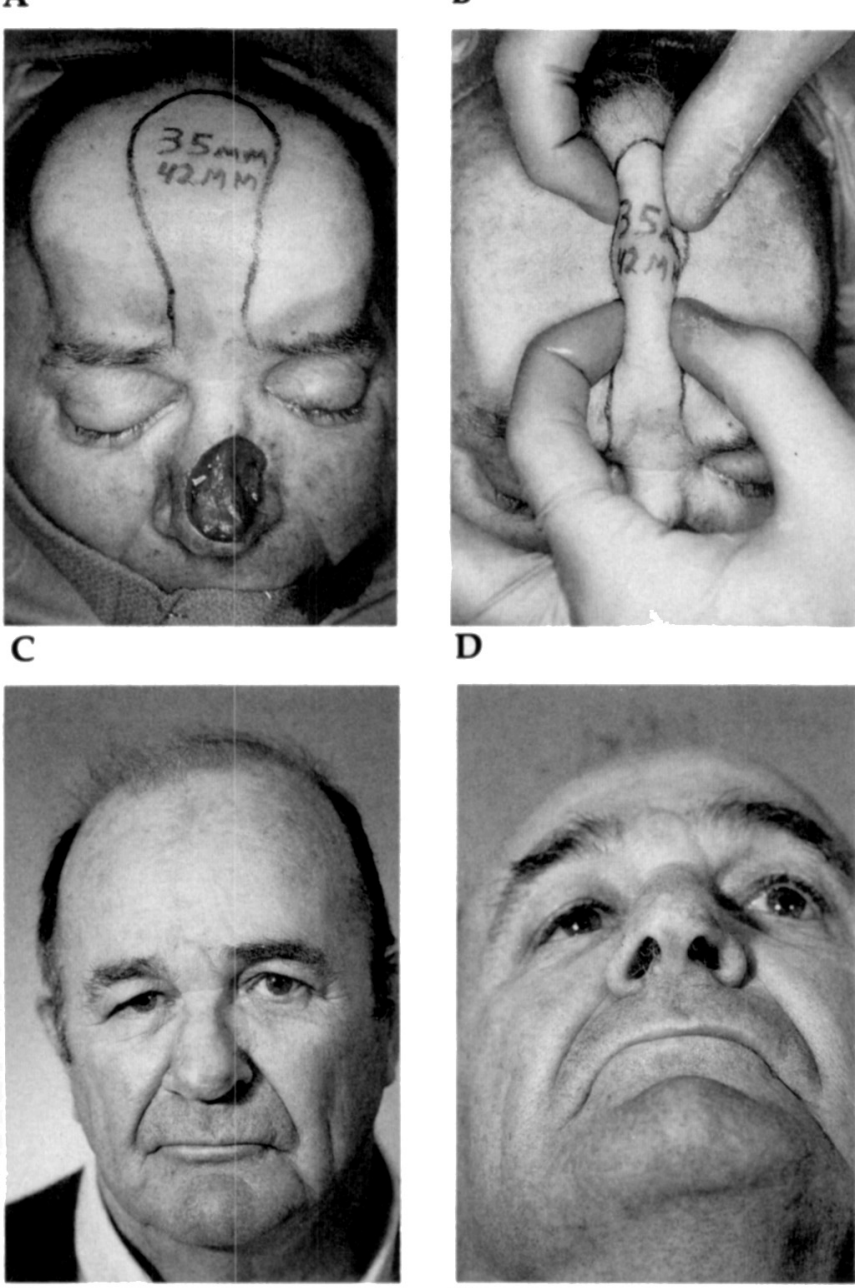

$\mathbf{E}$

Figure 5. $A$ and B) 4.0-cm wide nasal defect following excision of a basal cell carcinoma. C) Rapid intraoperative tissue expansion allowed stretching of a 3.5-cm wide midline forehead flap to $4.2 \mathrm{~cm}$. D) Stretching of flap and surrounding forehead skin facilitated wound closure without tension. E and $F$ ) Appearance of forehead and reconstructed nose 7 months following surgery.
Specifically, prolonged expansion can increase skin surface area approximately $135 \%$, compared with $30 \%$ for rapid expansion. ${ }^{7}$ In addition, the stretchback phenomenon probably reduces the skin surface area gained by rapid expansion to 10 to $15 \%$. Thus, this technique is helpful only in situations where repair of a wound can be accomplished with a local flap, but will be problematic because of excessive wound tension.

\section{Discussion}

Prolonged controlled tissue expansion offers the advantages of increasing locally available tissue with preservation of sensation and adnexal structures. Thus, the missing skin of the midfacial defect can be replaced with skin of similar color, thickness, and appendages (hair growth). This is unlike any other form of reconstruction because there is no need for a secondary defect unless creation of a flap is necessary. Expanded skin can simply be advanced into the defect for primary closure. In instances requiring the use of a flap, the secondary defect can be easily closed because of the additional skin created by the expansion process; thus, expansion obviates sacrificing cosmesis of one area to improve that of another.

The use of prolonged controlled skin expansion to close large defects of the midface and orbit usually eliminates the need for multiple flaps or grafting procedures, thus reducing the overall cost of reconstruction. Tissue expansion is tolerated well by most patients, can be performed in an outpatient setting, and can be accomplished prior to the ablative or reconstructive procedure so that additional tissue will be available at the time of excision and reconstruction.

Flaps harvested from prolonged controlled expanded skin have increased vascularity, ${ }^{5}$ thus flaps that would fail as simple primary procedures can be harvested from expanded skin with relative impunity, significantly improving the surgeon's flexibility during reconstructive procedures. The flexibility is enhanced because expansion can be repeated. That is, the tissue can be re-expanded a second or third time if necessary to achieve the final goals of reconstruction.

The major disadvantage of prolonged controlled tissue expansion in reconstruction of the midface is that it involves two surgical procedures; one to implant the expander and the other to remove the expander and perform reconstruction.

Tissue expansion is labor intensive in that frequent visits to the office are necessary for the inflation of the implant. This is obviated in some circumstances by training paramedical personnel in the patient's community or a patient's family member to carry out the actual inflation process. 
Another major disadvantage of tissue expansion in the head and neck region is the temporary visible deformity that results from the expander, particularly toward the end of the expansive process.

The advantages of prolonged controlled tissue expansion appear to outweigh the disadvantages when the surgeon is confronted with situations similar to those presented in this article in which there is an extensive midfacial defect and limited local tissue available for reconstruction. Prolonged controlled tissue expansion creates large amounts of additional skin for reconstructing midfacial defects, and eliminates the need for multiple, regional, distant or microsurgical flaps. On other occasions, there may be adequate tissue available to repair a midfacial defect with a local flap, but wound tension of either the donor site closure or the transposed flap may be excessive. In such situations, rapid intraoperative tissue expansion may assist in reducing this tension.

\section{References}

1. Baker SR, Swanson NA. Management of nasal cutaneous malignant neoplasms: an interdisciplinary approach. Arch Otolaryngol 1983;109:473-9.
2. Baker SR. Options for reconstruction in head and neck surgery. In: Cummings CW, Frederickson JM, Harker LA, Krause CJ, Schuller DE, eds. Otolaryngology-Head and Neck Surgery Update I. CV Mosby, St. Louis, 1989:192248.

3. Baker SR, Swanson NA. Tissue expansion of the head and neck-indications, techniques and complications. Arch Otolaryngol 1990;116:1143-53.

4. Baker SR, Swanson NA. Clinical applications of tissue expansion in head and neck surgery. Laryngoscope 1990; 100:313-9.

5. Baker SR, Swanson NA. Rapid intraoperative tissue expansion in reconstruction of the head and neck. Arch Otolaryngol 1990;116:1431-4.

6. Hoffman HT, Baker SR. Nasal reconstruction with the rapidly expanded forehead flap. Laryngoscope 1989;99: 1096-8.

7. Machida BK, Lie-Shindo M, Sadaki GH, Rich DH, Chandrasoma P. Immediate versus chronic tissue expansion. Presented at the Fifth International Symposium of Facial Plastic and Reconstructive Surgery, June 1989, Toronto, Canada. 